Research Paper

\title{
LINC01272/miR-876/ITGB2 axis facilitates the metastasis of colorectal cancer via epithelial-mesenchymal transition
}

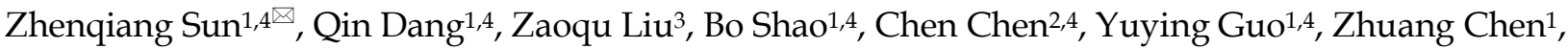 \\ Quanbo Zhou ${ }^{1}$, Shengyun $\mathrm{Hu}^{1}$, Jinbo Liu ${ }^{1 \otimes}$, Weitang Yuan ${ }^{1 凶}$ \\ 1. Department of Colorectal Surgery, The First Affiliated Hospital of Zhengzhou University, Zhengzhou 450052, Henan, China. \\ 2. School of Life Sciences, Zhengzhou University, Zhengzhou 450001, Henan, China. \\ 3. Department of Interventional Radiology, The First Affiliated Hospital of Zhengzhou, University, Zhengzhou, 450052, Henan, China. \\ 4. Academy of Medical Sciences, Zhengzhou University, Zhengzhou 450052, Henan, China. \\ Corresponding authors: Zhenqiang Sun, E-mail: fccsunzq@zzu.edu.cn; Weitang Yuan, E-mail: yuanweitang@zzu.edu.cn; Jinbo Liu, E-mail: \\ fccliujb@zzu.edu.cn; ORCID: Zhenqiang Sun, https:// orcid.org/0000-0001-5926-2716.
}

(1) The author(s). This is an open access article distributed under the terms of the Creative Commons Attribution License (https://creativecommons.org/licenses/by/4.0/). See http://ivyspring.com/terms for full terms and conditions.

Received: 2020.11.09; Accepted: 2021.04.26; Published: 2021.05.05

\begin{abstract}
Background: At the time of diagnosis, colorectal cancer (CRC) patients are usually in an advanced stage of disease, which is accompanied by metastasis. Long noncoding RNAs (IncRNAs) play critical regulatory roles in cancer biology. However, the contributions of IncRNA LINC01272 to CRC remain elusive.

Methods: Bioinformatics and the survminer $\mathrm{R}$ package were used to predict intermolecular correlations and prognostic indicators. Quantitative real-time PCR was used to examine molecular expression. In vitro experiments, including migration assays, invasion assays, and wound healing assays, were used to investigate the effects of LINC01272, ITGB2 and miR-876 on CRC cell migration and invasion abilities. Furthermore, a dual-luciferase reporter gene assay was performed to explore the potential mechanism by which LINC01272 contributes to CRC.

Results: We found that LINC01272 was highly expressed in multiple cancers and closely related to core epithelial-mesenchymal transition (EMT) factors and that high levels of LINC01272 are associated with a poor prognosis in CRC patients. QRT-PCR revealed that LINC01272 was highly expressed and negatively associated with miR-876 in CRC. Additionally, LINC01272 or ITGB2 silencing reduced, while miR-876 overexpression promoted, the invasiveness and metastatic capacity of CRC cells in vitro. Moreover, LINC01272 potentially targeted miR-876, and miR-876 potentially targeted ITGB2.

Conclusion: LINC01272 was highly expressed in CRC and predicted a poor prognosis. LINC01272 promoted EMT and metastasis by regulating miR-876/ITGB2 to act as an oncogene in CRC. LINC01272 may be a promising prognostic biomarker and therapeutic target for the treatment of CRC patients in the future.
\end{abstract}

Key words: colorectal cancer; long noncoding RNA; LINC01272; EMT; metastasis

\section{Introduction}

Colorectal cancer (CRC) is the third most common type of cancer and the third leading cause of cancer-related death worldwide [1]. The overall 5 -year survival rate of patients with CRC is $65 \%$. Approximately $12 \%$ of individuals under 50 years of age will be newly diagnosed with CRC [2]. Therefore, robust diagnostic, prognostic and predictive biomarkers are clearly and urgently needed to detect advanced colon polyps and early-stage CRC, which are the forms of CRC that are most effectively treated with the current therapies available to specific CRC patients. The last two decades of research have demonstrated the potential of long noncoding RNAs to be used as biomarkers of CRC [3, 4]. Continued investigation of this promising class of biomarkers is likely to lead to high-efficiency assays that can be used to prevent and manage CRC in patients [5]. LncRNAs are a class of RNA molecules defined as transcripts longer than 200 nucleotides that lack protein coding potential [6]. Increasing evidence 
suggests that lncRNAs may mediate oncogenic or anti-oncogenic effects and may be a new class of cancer biomarkers and therapeutic targets [7-9]. Haberman $Y$ et al. reported that LINC01272 upregulation was significantly associated with mucosal damage in early-onset Crohn's disease (CD) [10]. In CD, upregulated LINC01272 was associated with a myeloid proinflammatory signature and regulated this signature in response to inflammatory signals. LINC01272 was also reported to be significantly upregulated in tissue and plasma samples from patients with inflammatory bowel disease (IBD) compared with those from healthy controls. Correspondingly, LINC01272 was considered a potential novel diagnostic biomarker of IBD [11]. LINC01272 is expected to be used as a potential diagnostic biomarker of gastric cancer that acts by regulating the expression of EMT-related proteins to promote the metastatic ability of gastric cancer cells [12]. Nevertheless, the role of LINC01272 in CRC remains elusive.

Therefore, we aimed to further explore the effect of LINC01272 in order to assess whether LINC01272 can become a useful diagnostic and prognostic biomarker and an important therapeutic target for the treatment of CRC patients. The expression patterns of LINC01272 in CRC cancer tissues were analysed, and its biological function in colon cancer cells was investigated to reveal a tumour biomarker and therapeutic target gene in CRC.

\section{Materials and methods}

\section{Tissue specimen and clinical data collection}

This study was approved by the First Affiliated Hospital of Zhengzhou University. A total of 27 paired CRC tissues and matched adjacent non-tumour tissues were obtained from patients who underwent surgical resection at The First Affiliated Hospital of Zhengzhou University. None of the patients received any preoperative chemotherapy or radiotherapy. Written informed consent was obtained from all the patients. The inclusion criteria were as follows: no preoperative chemotherapy, radiotherapy, or targeted therapy; no other types of tumours; and no autoimmune diseases. The specimens obtained during surgery were immediately snap frozen in liquid nitrogen and stored at $-80{ }^{\circ} \mathrm{C}$ until RNA extraction. Clinical staging of the specimens was based on the NCCN (2019) guidelines.

\section{Bioinformatic prediction}

RNA-Seq (FPKM normalized) data were retrieved from the TCGA portal, the Kaplan-Meier method was used to assess the prognostic significance of LINC01272, and the optimal cut-off value was determined by the survminer $\mathrm{R}$ package (Figure S1). The RNA-Seq datasets in GEPIA (http://gepia. cancer-pku.cn/) were used to analyse the expression and prognostic relevance of LINC01272, ITGB2, and EMT core factors. The potential role of LINC01272 in targeting miR-876 was predicted using DIANA tools (http://carolina.imis.athena-innovation.gr/diana tools/). The potential role of miR-876 in targeting ITGB2 was predicted through the miRbase, miRecords and TargetScan databases.

\section{RNA extraction and reverse transcription}

Total RNA was isolated from CRC tissues, paired adjacent non-cancerous tissues and CRC cells with RNAiso Plus reagent (Takara, Dalian, China), according to the manufacturer's instructions. The RNA quality was evaluated using a NanoDrop One C (Waltham, MA, USA), and the RNA integrity was assessed using agarose gel electrophoresis. An aliquot of $1 \mu \mathrm{g}$ of total RNA was reverse-transcribed into complementary DNA (cDNA) using a High-capacity cDNA Reverse Transcription kit (TaKaRa Bio, Japan), according to the manufacturer's protocol. miRNAs were reverse transcribed using a miRNA reverse transcription kit (TaKaRa Bio, Japan).

\section{Quantitative real-time PCR}

Quantitative real-time PCR (qRT-PCR) was performed using SYBR Assay I Low ROX (Eurogentec, USA) and SYBR ${ }^{\circledR}$ Green PCR Master Mix (Yeason, Shanghai, China) to detect gene expression. The $2^{-\Delta \Delta C t}$ method was used to calculate the relative levels of gene and miRNA expression. The primers are listed in Table S1. GAPDH or U6 was used as the endogenous control for normalization. qRT-PCR assays were performed in triplicate with the following conditions: (1) $95{ }^{\circ} \mathrm{C}$ for $5 \mathrm{~min}$ and (2) 40 cycles of $95^{\circ} \mathrm{C}$ for $10 \mathrm{~s}$ and $60^{\circ} \mathrm{C}$ for $30 \mathrm{~s}$. The relative expression of LINC01272 was calculated using the $\triangle \mathrm{CT}$ (Ct lncRNA-Ct GAPDH) method. miRNA qPCR was carried out according to a miRNA qPCR kit (TaKaRa Bio, Japan), and U6 was used as the internal reference.

\section{Cell culture}

The HCT116 human colon cancer cell line was purchased from the Shanghai Institute of Cell Biology, Chinese Academy of Sciences (Shanghai, China). The HCT116 cells were cultured in high-glucose DMEM (Sigma, D6429) containing 10\% FBS (Biological Industries, Kibbutz Beit Haemek, Israel) and incubated at $37^{\circ} \mathrm{C}$ in $5 \% \mathrm{CO} 2$.

\section{siRNA transfection of colon cancer cells}

Silencer select small interfering RNAs (siRNAs) specific for LINC01272 and a control siRNA were 
obtained from RiboBio (Guangzhou, China). To silence RNA molecules in colon cancer cells, LINC01272-specific siRNA (si-h-LINC01272, target sequence: GGAGAATAAACCCTCGGAT), ITGB2specific siRNA (si-h-ITGB2, target sequence: GTACA AGAGGAGCAACGAA) and control siRNA were transfected into HCT116 cells. The transfection was conducted with Lipofectamine 3000 (Thermo Fisher, L3000-015), according to the manufacturer's instructions.

\section{Invasion and migration assays}

To assess the migratory ability of the transfected cells, the cells were seeded in 24-well plates (Corning, NY, USA) and incubated under permissive conditions until they reached $90 \%$ confluence. After serum starvation for $24 \mathrm{~h}$, wounds were created in the confluent cells with a $20-\mu \mathrm{L}$ pipette tip. Then, the scraped cells were lightly washed with PBS, and serum-free medium was added to the 24-well plates. Wound healing within the scrape was then observed and photographed at the indicated time points, and the area was analysed by ImageJ (version 1.30, free at http://rsb.info.nih.gov/ij/).

According to the experimental objective of analysing invasion or migration, Transwell chambers (Corning, NY, USA) were prepared with or without Matrigel. Then, blood serum medium (10\% FBS) was added to the lower chamber. After transfection with si-LINC01272 and inhibitor control, HCT116 cells were digested to prepare a cell suspension, and this suspension was added to the upper chamber and incubated for $24 \mathrm{~h}$. At the end of this incubation, the residual cells in the upper chamber were gently wiped with cotton swabs. The cells were fixed with $4 \%$ paraformaldehyde and stained with $1 \%$ crystal violet for $30 \mathrm{~min}$. After washing three times with PBS, the cells were imaged and counted with an OLYMPUS FV1000 confocal microscope. Every experiment was performed three times for statistical analysis.

\section{Dual-luciferase reporter assay}

Luciferase activity assays were performed with the Dual-Luciferase Reporter Assay System (Promega, Madison, WI, USA). Putative wild-type (WT) and mutant (Mut) miR-876-5p-binding sites in the 3'-UTR of LINC01272 or ITGB2 mRNA, termed LINC01272Luc WT or LINC01272-Luc MT and ITGB2-Luc WT or ITGB2-Luc MT, were cloned into the psiCHECK2 vector (Promega, Madison, WI, USA). HCT116 cells were transfected with miR-876 mimics, the Renilla luciferase reporter vector (Promega), and the LINC01272-Luc WT, LINC01272-Luc MT, ITGB2-Luc WT or ITGB2-Luc MT reporter constructs using Lipofectamine 3000 reagent (Thermo Fisher,
L3000-015), according to the manufacturer's instructions. As the internal control, $5 \mathrm{ng} /$ well Renilla luciferase plasmid was used. After $48 \mathrm{~h}$ of transfection, the firefly and Renilla luciferase activities were measured using the Dual-Luciferase Reporter Assay System. Correction for differences in transfection efficiency was performed by normalizing the firefly luciferase activity to the total Renilla luciferase activity.

\section{Statistical analyses}

All the statistical analyses were performed using SPSS version 24.0 (SPSS, Chicago, IL, USA) and GraphPad Prism 5.0 software (CA, USA). All the measurements were conducted in triplicate. Significant differences between the LINC01272 expression levels were analysed using two-sided Student's t-test. The indicated $\mathrm{P}<0.05$ was considered statistically significant.

\section{Results}

\section{LINC01272 was highly expressed in colorectal cancer}

We first examined the expression of LINC01272 in the normal and tumour groups via pan-cancer analysis. The expression data were retrieved from the UCSC portal. The analysis results showed that LINC0172 was highly expressed in multiple cancers, including colon adenocarcinoma (COAD), oesophageal carcinoma (ESCA), glioblastoma multiforme (GBM), head and neck squamous cell carcinoma (HNSC), kidney renal clear cell carcinoma (KIRC), kidney renal papillary cell carcinoma (KIRP), liver hepatocellular carcinoma (LIHC), lung adenocarcinoma (LUAD), lung squamous cell carcinoma (LUSC), pancreatic adenocarcinoma (PAAD), pheochromocytoma and paraganglioma (PCPG), rectum adenocarcinoma (READ), stomach adenocarcinoma (STAD), thyroid carcinoma (THCA) and uterine corpus endometrial carcinoma (UCEC) (Figure 1a, b). In addition, we further analysed the expression of LINC01272 in 27 pairs of CRC tissues and adjacent normal mucosal tissues using qRT-PCR, and the results showed that LINC01272 was prominently overexpressed in colorectal cancer $(\mathrm{P}<$ 0.001) (Figure 1c), indicating that LINC01272 may facilitate CRC carcinogenesis. We also explored LINC01272 expression in different CRC cells and found that LINC01272 was highly expressed in SW480, HT29, LOVO and HCT116 cells compared with normal intestinal mucosa cells (NCM460). LINC01272 was the most highly expressed in HCT116 cells, which were the cells most easily transfected with 
plasmids (Figure 1d). Furthermore, we performed the following in vitro assays in HCT116 cells.

\section{High expression of LINCO1272 predicted poor prognosis of CRC patients}

The prognostic value of lncRNA expression was evaluated by the survminer $\mathrm{R}$ package and showed that the overall survival (OS) rate of COAD patients with high LINC01272 expression was higher than that of COAD patients with low LINC01272 expression (122 patients, $\mathrm{P}<0.05$ ) (Figure 2a). The OS and disease-free survival (DFS) rates of READ patients with high LINC01272 expression were higher than those of READ patients with low LINC01272 expression (32 patients, $\mathrm{P}<0.05$ ) (Figure $2 \mathrm{~b}$ ). Therefore, LINC01272 has promising prognostic value in patients with CRC.

\section{Both LINC01272 and ITGB2 were closely associated with core EMT molecules in CRC}

Correlation analysis was carried out with the RNA-Seq GEPIA dataset. The results demonstrated that LINC01272 expression was positively associated with VIM (Vimentin), CDH2 (N-cadherin), SNAI2
(SLUG), SNAI1 (SNAI), TWIST1 (TWIST), ZEB1, and ZEB2 (all $\mathrm{P}<0.001$ ) and negatively associated with CDH1 (E-cadherin) $\quad(\mathrm{P}<0.05) \quad$ (Figure $3 a) . \quad$ ITGB2 expression was positively associated with VIM (Vimentin), CDH2 (N-cadherin), SNAI2 (SLUG), SNAI1 (SNAI), TWIST1 (TWIST), ZEB1, and ZEB2 (all $\mathrm{P}<0.001)$ and negatively associated with CDH1 (E-cadherin) $\quad(\mathrm{P}<0.01) \quad$ (Figure 3b). Thus, both LINC01272 and ITGB2 were closely associated with EMT in CRC.

\section{LINC01272 was closely associated with ITGB2 in CRC}

The relationship between LINC01272 and ITGB2 was analysed with the RNA-Seq dataset GEPIA, and the results showed that LINC01272 was positively associated with ITGB2 in colon cancer, rectal cancer and CRC $(\mathrm{P}<0.001)$ (Figure 4a, b, c). Additionally, ITGB2 and LINC01272 were detected by qRT-PCR assay in 27 pairs of CRC samples. Correlation analysis was performed and showed that LINC01272 was positively associated with ITGB2 ( $\mathrm{P}<0.05)$ (Figure 4d).
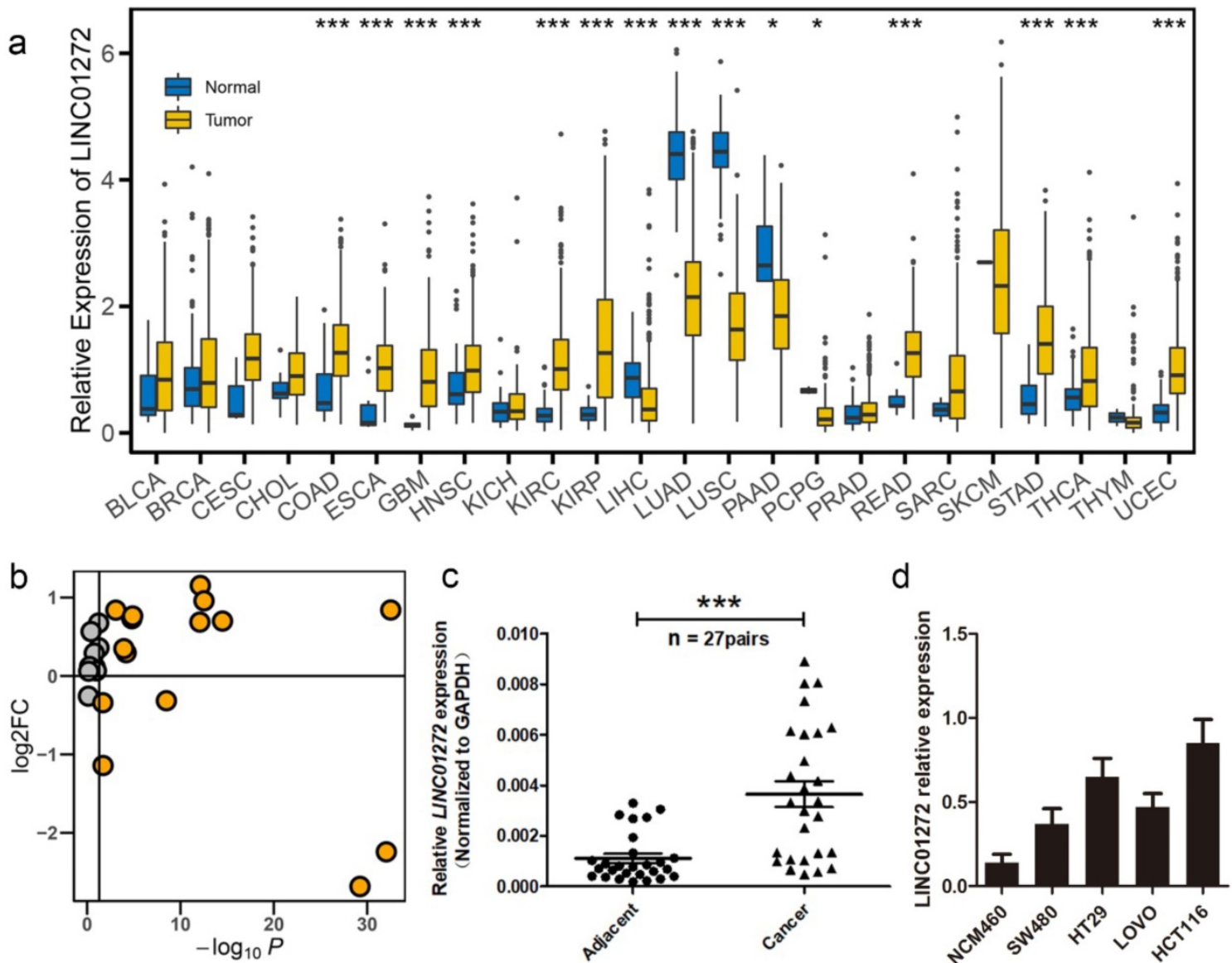

Figure 1. LINC01272 was highly expressed in CRC. a, LINC01272 expression in the normal and tumour groups in pan-cancer analysis. The expression data were retrieved from the UCSC portal $(* \mathrm{P}<0.05$, $* * \mathrm{P}<0.01$ and $* * * \mathrm{P}<0.001)$. $\mathbf{b}$, The log2FC and $\mathrm{P}$-value distribution of LINC01272 between the normal and tumour groups in pan-cancer analysis. LINC01272 was upregulated in a variety of tumours, and in 10 of these tumours, the upregulation was significant. c, In 27 paired human colon cancer tissues and adjacent non-cancerous tissues, LINC01272 was more highly expressed in tumour tissues than in normal adjacent normal tissues (P<0.001). d, LINC01272 showed higher expression in multiple CRC cells (SW480, HT29, LOVO, and HCT116) than in normal intestinal mucosa cells (NCM460). 

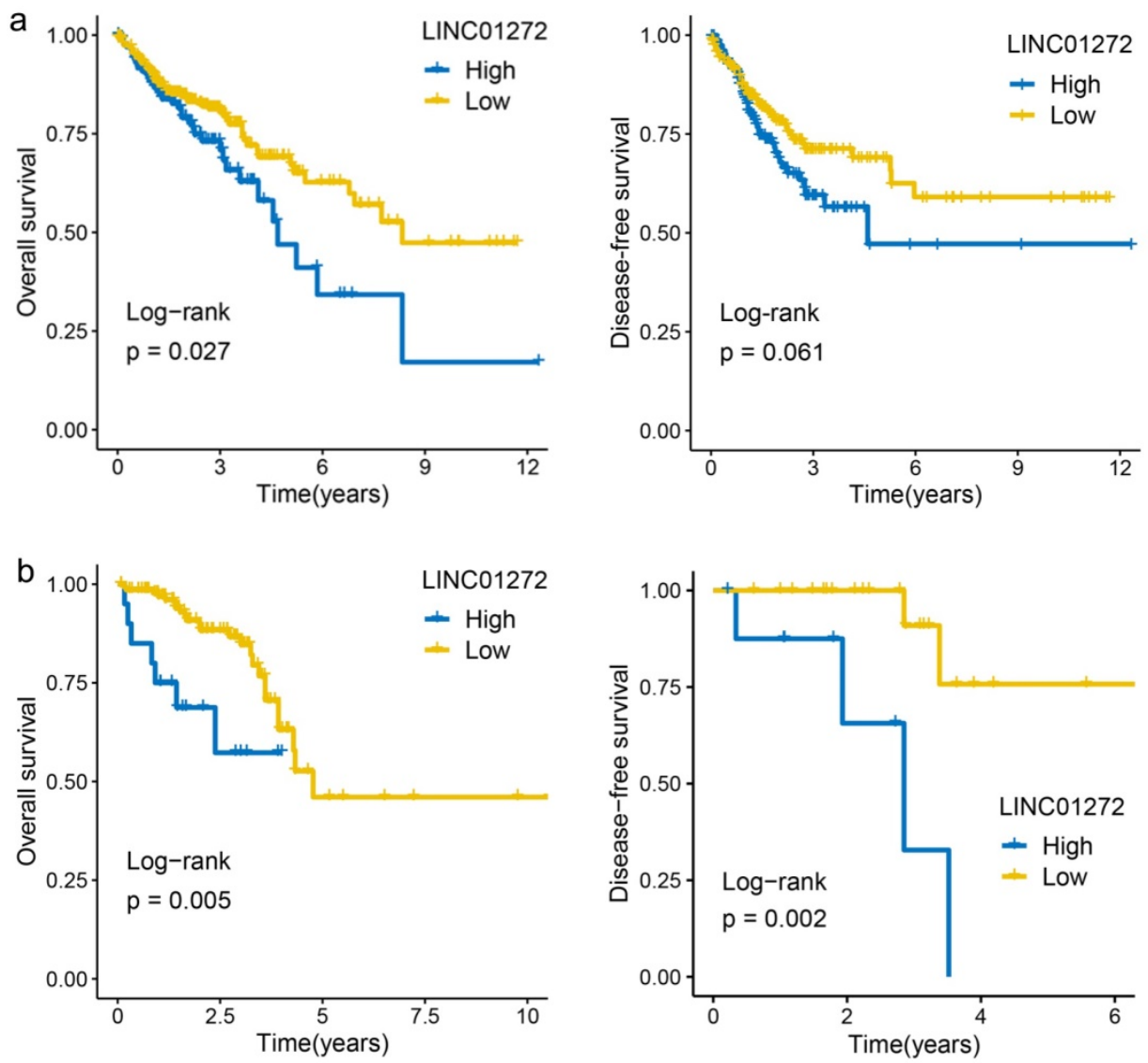

Figure 2. High expression of LINC01 272 predicts poor prognosis of CRC patients. a, The OS rate of colon cancer patients with high LINC01272 expression was higher than that of patients with low LINC01272 expression $(P<0.05)$, and the DFS showed valuable trends. b, The OS and DFS rates of rectal cancer patients of high LINC01272 expression were higher than those of rectal cancer patients with low LINC01272 expression $(\mathrm{P}<0.05)$.

\section{LINC01 272, miR-876 and ITGB2 regulated invasion and migration in vitro}

To explore the roles of LINC01272, miR-876 and ITGB2 in CRC cells, HCT116 cells were transfected with LINC01272 siRNA (si-LINC01272), ITGB2 siRNA (si-ITGB2), miR-876 mimics and their corresponding controls. The knockdown and overexpression efficiencies were confirmed via qRT-PCR analysis (Figure S2). Transwell assays were carried out and showed that by inhibiting LINC01272 or ITGB2 expression, miR-876 overexpression inhibited the migration and invasion of HCT116 cells (Figure 5a, b). Similarly, a wound healing assay further verified that by inhibiting LINC01272 or ITGB2 expression, miR-876 overexpression reduced the migration and motility of HCT116 cells (Figure 5c, d). Accordingly, LINC01272, miR-876 and ITGB2 could promote the invasion and migration of CRC cells.

\section{LINC01 272 bound to miR-876 and miR-876 targeted ITGB2 in CRC}

To study the mechanism underlying the effect of LINC01272, bioinformatic prediction was conducted via the DIANA lab database. The prediction analysis results showed that LINC01272 had a miR-876-binding sequence (Figure 6a). Based on the prediction results, a LINC01272-Luc WT plasmid and LINC01272-Luc MT plasmid were constructed. In addition, bioinformatic prediction with rstudio (version 1.0.136) and the $\mathrm{R}$ package revealed a negative correlation between LINC01272 and miR-876 in CRC (Figure 6b). qRT-PCR was performed to analyse 15 CRC samples. Correlation analysis showed that the LINC01272 expression levels were negatively associated with the miR-876 expression levels in CRC $(\mathrm{P}<0.05)$ (Figure 6c). The LINC01272-Luc WT plasmid, LINC01272-Luc MT plasmid and their corresponding controls were transfected into HCT116 cells. Transwell assays were carried out and showed that miR-876 inhibited the luciferase activity of the LINC01272-Luc WT plasmid but not the luciferase activity of the LINC01272-Luc MT plasmid in HCT116 cells (Figure 6e). Moreover, bioinformatic prediction via prediction analysis of the miRbase, miRecords and TargetScan databases showed that the ITGB2 3'UTR contained a miR-876-binding sequence (Figure 6d). A ITGB2-Luc WT plasmid and ITGB2-Luc MT plasmid were constructed. Similarly, Transwell assays were 
performed and showed that miR-876 inhibited the luciferase activity of the ITGB2-Luc WT plasmid but not the luciferase activity of the ITGB2-Luc MT plasmid in HCT116 cells (Figure 6e). Consequently, LINC01272 might promote ITGB2 expression by targeting miR-876 in CRC.

\section{LINC01 272/miR-876/ITGB2 axis regulated EMT and metastasis}

To verify the effects of LINC01272, miR-876 and ITGB2 on EMT in CRC, HCT116 cells were transfected with si-LINC01272, si-ITGB2, miR-876 mimics and their corresponding controls. After culturing for 48 hours, RNA was extracted for qRT-PCR assay. The results showed that by inhibiting LINC01272 or ITGB2 expression, miR-876 overexpression inhibited the expression of EMT core factors, including VIM, E-cadherin, SLUG, TWIST and ZEB1 (Figure 7a). In brief, LINC01272 enhanced the EMT process by regulating ITGB2-miR-876, consequently promoting the invasion and metastasis of CRC (Figure 7b).
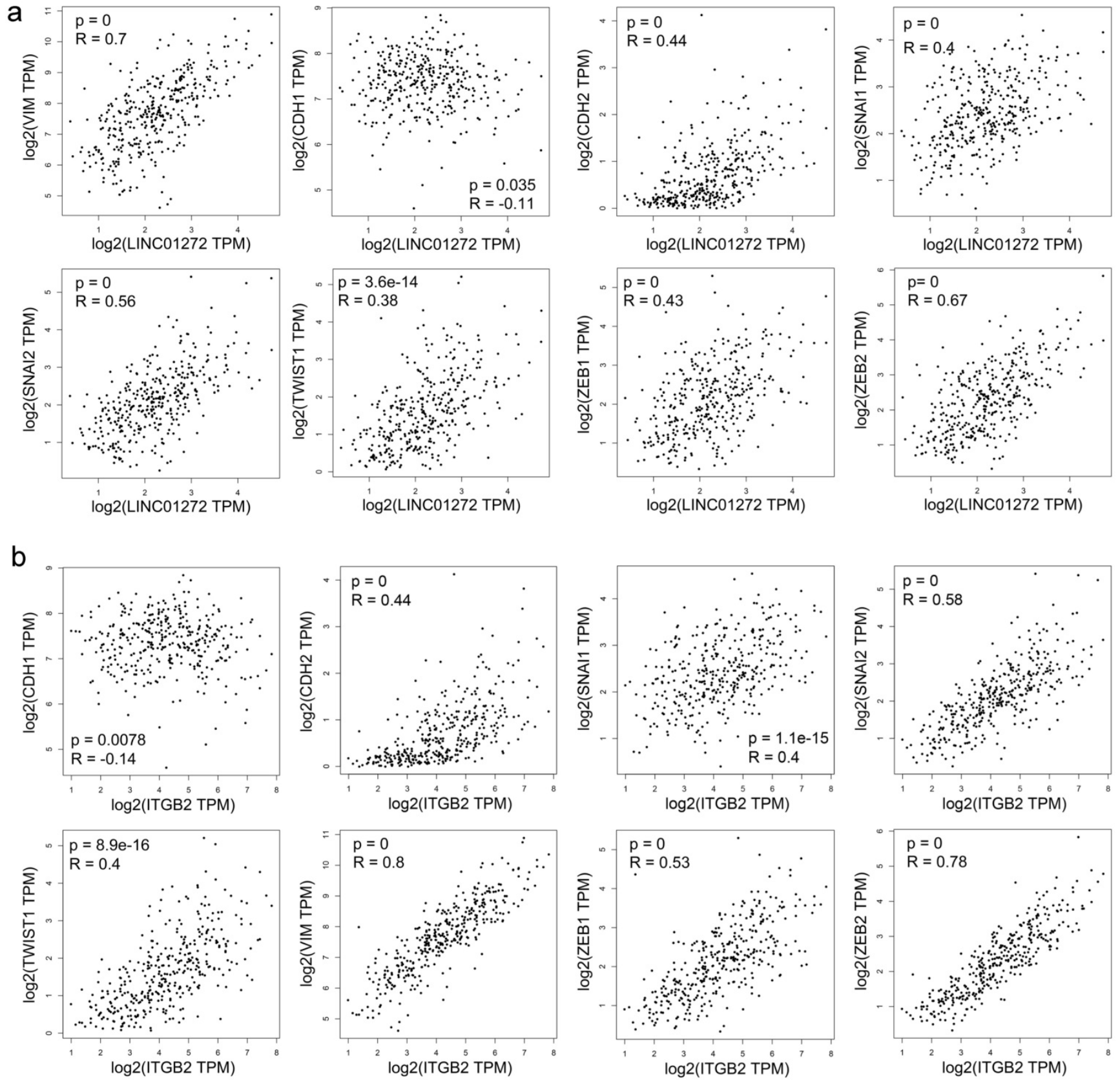

Figure 3. Both LINC01272 and ITGB2 were closely associated with core EMT molecules in CRC. a, LINC01272 expression was positively associated with VIM (Vimentin), CDH2 (N-cadherin), SNAI2 (SLUG), SNAII (SNAI), TWIST1 (TWIST), ZEB1, and ZEB2 (all $\mathrm{P}<0.001$ ) and negatively associated with CDHI (E-cadherin) (P<0.05). b, ITGB2 expression was positively associated with VIM (Vimentin), CDH2 (N-cadherin), SNAI2 (SLUG), SNAI1 (SNAI), TWIST1 (TWIST), ZEB1, and ZEB2 (all P<0.001) and negatively associated with $\mathrm{CDH1}(\mathrm{E}$-cadherin) $(\mathrm{P}<0.01)$. 

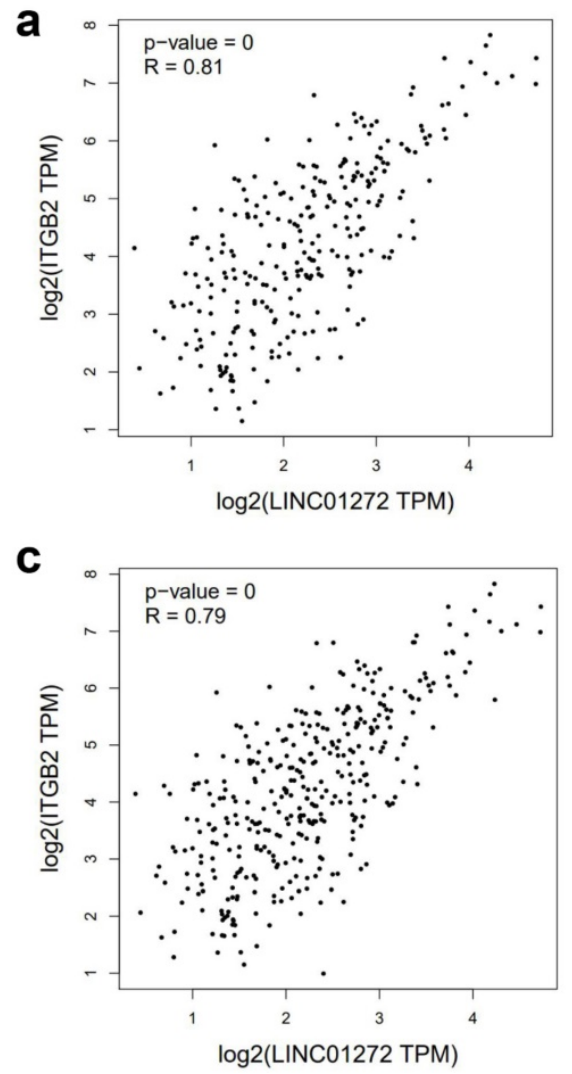

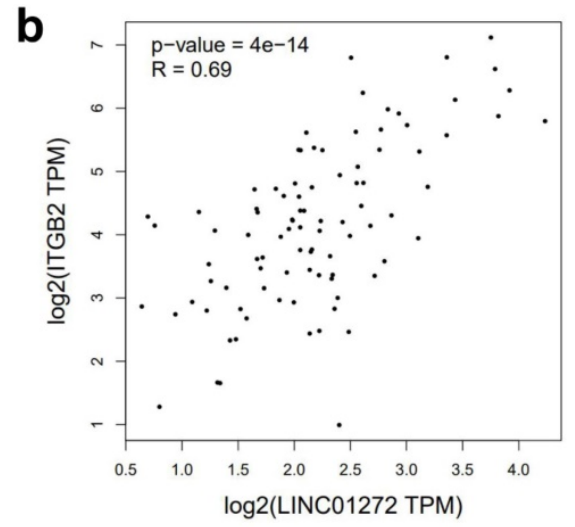

d

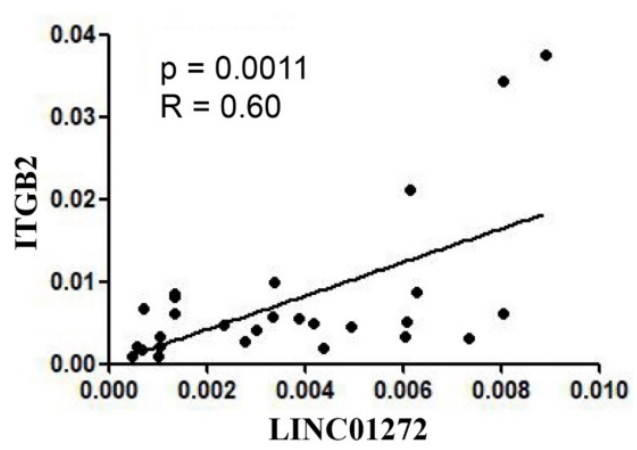

Figure 4. LINC01272 was closely associated with ITGB2 in CRC. a, b and c, LINC01272 was positively associated with ITGB2 in CRC based on analysis of the RNA-Seq dataset GEPIA (all P<0.001). d, In 27 paired human CRC tissues, LINC01272 was considerably positively associated with ITGB2, according to $\mathrm{qRT}$-PCR assay (P<0.05).

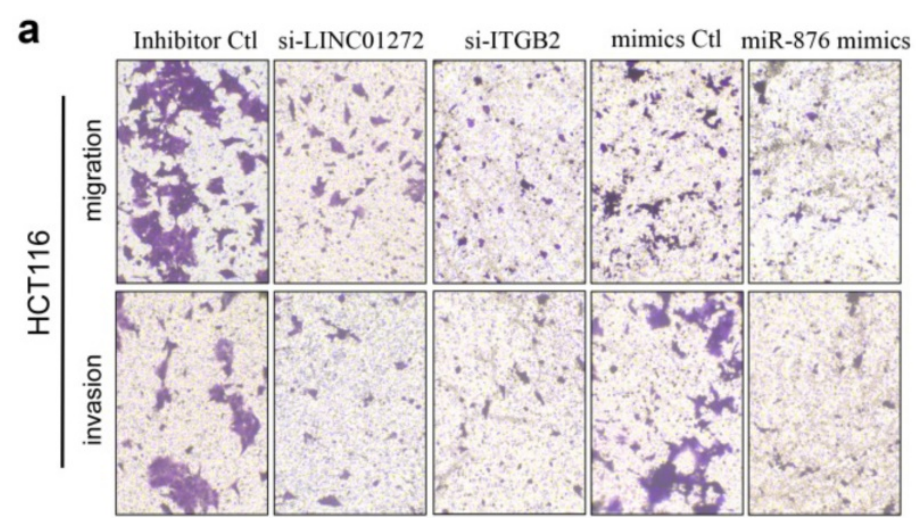

C

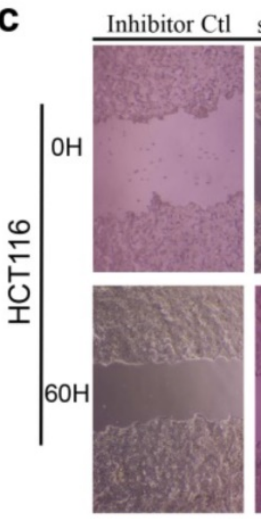

b
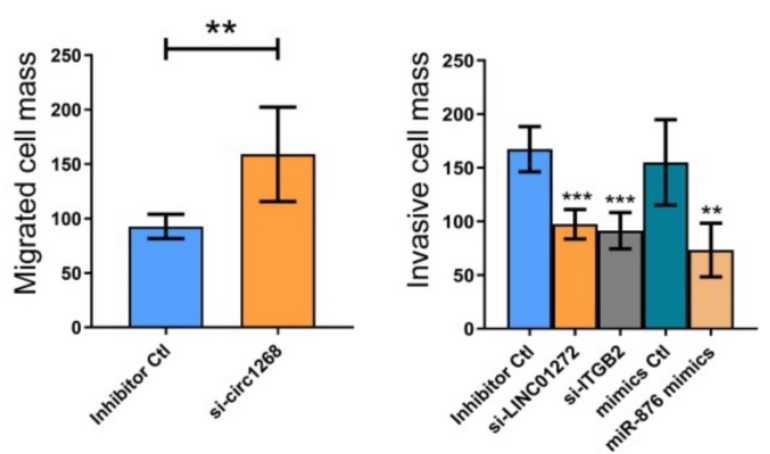

Figure 5. LINC01272, miR-876 and ITGB2 regulated invasion and migration in vitro. For the Transwell assay, HCT116 cells were transfected with si-LINC01272, si-ITGB2, miR-876 mimics and their corresponding controls. $\mathbf{a}$ and $\mathbf{b}$, Inhibition of LINC01272 or ITGB2 expression or overexpression of miR-876 impaired the migration and invasion of HCT11 16 cells. $c$ and d, Wound healing assay showed that down-regulation of LINC01272 or ITGB2 weakened the migration and motility of HCT116 cells. *p $<0.05$, **p $<0.01$ and $* * * \mathrm{p}<0.001$ compared to the control group by Student's t-test. 
a

LINC01272 MT 5' AGUCUCUUCCCUGGUCUUCCGACGUAAGC 3'

LINC01272 WT 5' AGUCUCUUCCCUGGUCUUCCAGAAAUCCC 3'

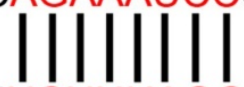

b

3' ACCACUAAGUGUUUUCUUUAGGU 5' miR-876
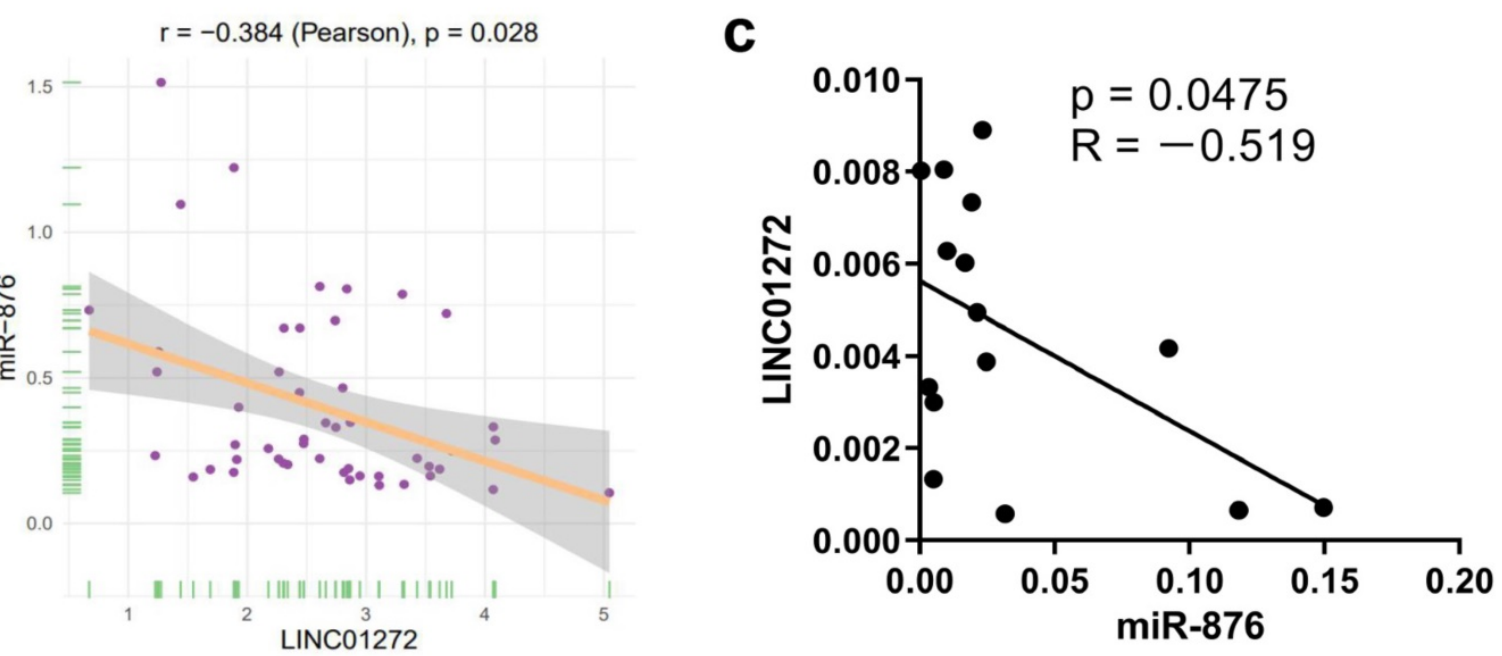

d ITGB2 MT 5' CACCAAUUAACCUAGCCAAGC 3'

ITGB2 WT 5' CACCAAUUAACCAGAAAUCCA 3'

\| $\mid\|\| \|$

3' ACCACUAAGUGUUUCUUUAGGU 5' miR-876
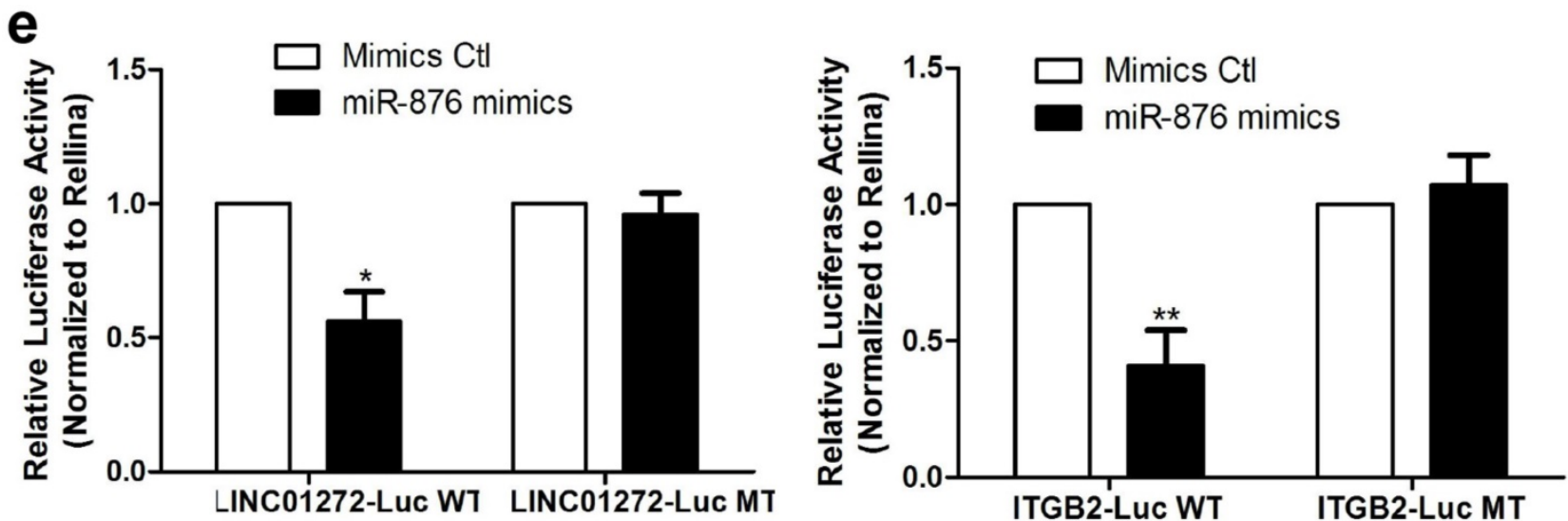

Figure 6. LINC01 272 bound to miR-876 and miR-876 targeted ITGB2 in CRC. a, Prediction analysis with the DIANA lab database showed that LINC01272 contained a miR-876-binding sequence. Based on the above, a LINC01272-Luc WT plasmid and LINC01272-Luc MT plasmid were constructed. b and c, Bioinformatics and qRT-PCR analyses were performed to detect LINC01272 and miR-876 and showed that these two factors were negatively associated $(P<0.05)$. $d$, Based on prediction analysis of the miRbase, miRecords and TargetScan databases, the ITGB2 3'UTR contained a miR-876-binding sequence. e, A LINC01272-Luc WT plasmid, LINC01272-Luc MT plasmid and their corresponding controls were transfected into HCT116 cells. Transwell assays were carried out and showed that miR-876 inhibited the luciferase activity of the LINC01272-Luc WT plasmid but not the luciferase activity of the LINC01272-Luc MT plasmid in HCT116 cells. Similarly, Transwell assays were performed and showed that miR-876 inhibited the luciferase activity of the ITGB2-Luc WT plasmid but not the luciferase activity of the ITGB2-Luc MT plasmid in HCT116 cells. Three independent experiments were performed. ${ }^{*} \mathrm{p}<0.05,{ }^{* *} \mathrm{p}<0.01$ compared to the control group by Student's t-test.

\section{Discussion}

CRC is the $3^{\text {rd }}$ most commonly diagnosed cancer in males and the $2^{\text {nd }}$ most commonly diagnosed cancer in females worldwide [13]. Cancer metastasis is a process during which cancer cells disseminate from the primary tumour to distant organs. Additionally, the CRC patient population as a whole is rapidly shifting toward a younger age range [2]. Therefore, the identification of novel biomarkers of advanced CRC is urgently needed to achieve early diagnosis and establish effective strategies with precise targets for improving patient prognosis. 


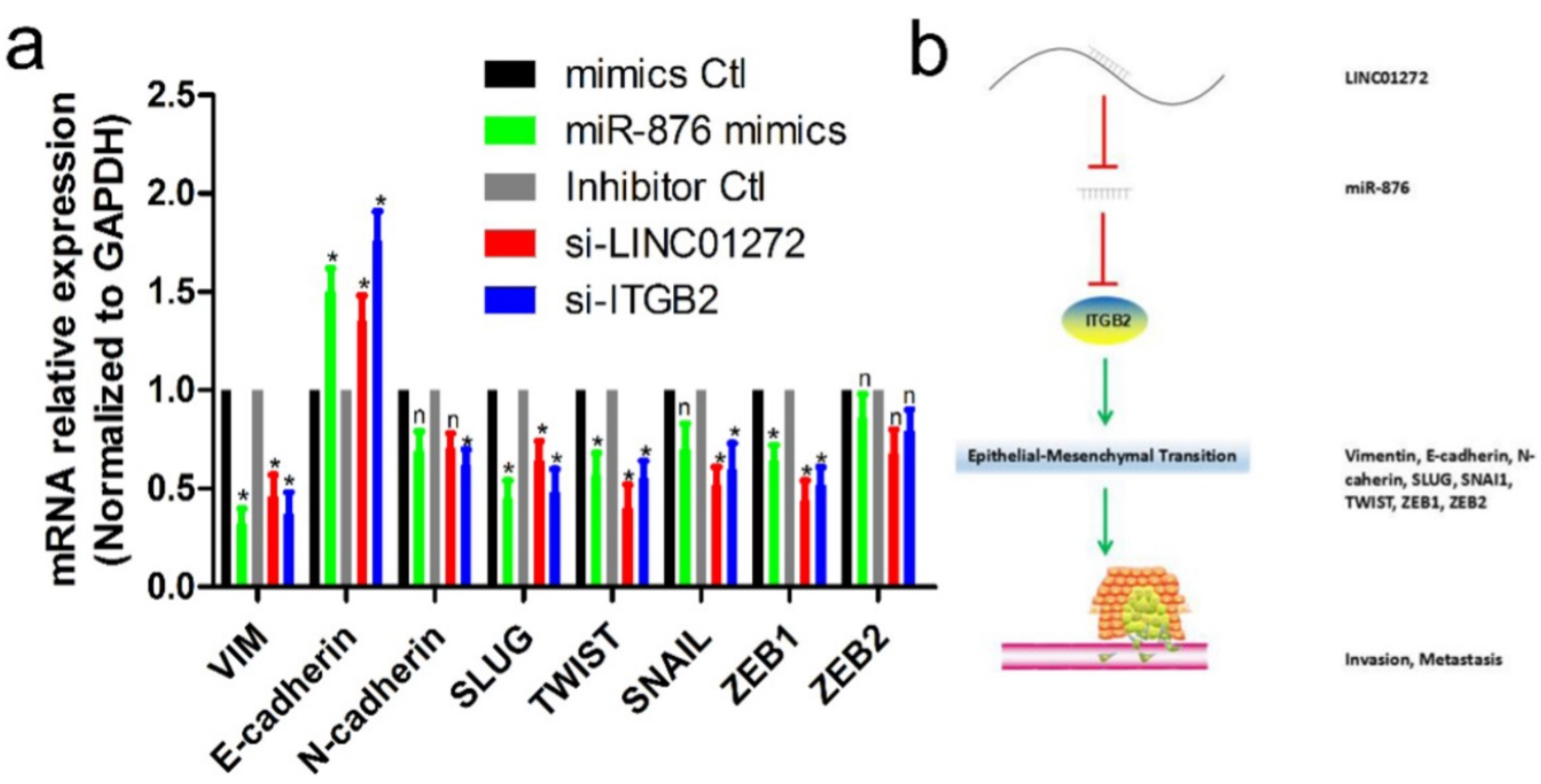

Figure 7. The LINC01272/miR-876/ITGB2 axis regulated EMT and metastasis. a, HCT116 cells were transfected with si-LINC01272, si-ITGB2, miR-876 mimics and their corresponding controls. After culturing for 48 hours, RNA was extracted for qRT-PCR assay. The results showed that by inhibiting LINC01272 or ITGB2 expression, miR-876 overexpression inhibited the expression of EMT core factors, including VIM, E-cadherin, SLUG, TWIST and ZEBI. b, Schematic representation of a model depicting the major molecular mechanisms of the LINC01272/miR-876/TTGB2 axis in CRC.

Increasing studies have reported that lncRNAs have high tissue specificity and are expected to be promising drivers and biomarkers of various malignancies [14-18]. LncRNAs derived from conserved genomic locations play crucial biological roles with conserved functionality during embryonic development [19]. A large body of evidence has revealed the essential role of lncRNAs in all stages of angiogenesis and metastasis. For instance, we previously verified that downregulation of the long noncoding RNA ANRIL suppressed lymphangiogenesis and lymphatic metastasis in colorectal cancer [20]. YAP1-induced MALAT1 could promote EMT and angiogenesis by sponging miR126-5p in CRC [21]. RP11 was aberrantly expressed at low levels in CRC, and its low expression was closely associated with aggressive clinicopathological features and unfavourable prognosis in CRC patients [22]. Additionally, many previous studies have reported that lncRNAs could act as oncogenes or antioncogenes. The long noncoding RNA TTTY15, which is located on the $\mathrm{Y}$ chromosome, promoted prostate cancer progression by sponging let-7 [23]. LNMAT1 promoted the lymphatic metastasis of bladder cancer via CCL2-dependent macrophage recruitment [24]. LncGata6 maintained the stemness of intestinal stem cells and promoted intestinal tumorigenesis [25]. Downregulation of DGCR5 contributed to cervical cancer progression by activating Wnt signalling [26].

In our study, we first showed that LINC01272 was highly expressed in CRC tissues compared with normal colorectal tissues via an analysis of the GEPIA database. To the best of our knowledge, our study is the first to explore the biological effect of LINC01272 and demonstrate a significant association between LINC01272 and CRC prognosis. Further analysis showed that LINC01272 was closely associated with EMT-related molecules, such as VIM, CDH1, CDH2, SNAIL1, SNAI2, TWIST, ZEB1, and ZEB2. Extensive gene screening showed that ITGB2 had simultaneously negative correlations with these molecules. Currently, the role of ITGB2 remains unknown in CRC. In this study, ITGB2 was found to upregulate in CRC and positively correlate with LINC01272 expression. Our results also demonstrated that ITGB2 was involving in the proliferation, migration, and invasion of CRC. In order to further explore the potential relationship between LINC01272 and ITGB2, we performed bioinformatic predictions through the DIANA laboratory database. The results displayed that LINC01272 had binding sequences with miR-876. Further investigation revealed miR-876 displayed the inverse phenomenon relative to ITGB2. Thus, we hypothesized that a LINC01272/miR-876/ ITGB2 axis might be involved in EMT process of CRC. Subsequently, dual-luciferase reporter assays confirmed that miR-876 had potential binding capacities with LINC01272 and ITGB2. Transwell and wound healing assays further verified that LINC01272/miR-876/ITGB2 axis could regulate invasion and migration of tumor cells. Overall, we reported that LINC01272/miR-876/ITGB2 axis might facilitate the metastasis of CRC via EMT process. 
In conclusion, our study provides solid evidence that LINC01272 was highly expressed in CRC and that high LINC01272 expression predicted a poor prognosis. LINC01272 favoured EMT and metastasis by regulating miR-876/ITGB2 to act as an oncogene in CRC. LINC01272 will potentially become a prognostic biomarker and a therapeutic target for the treatment of CRC patients in the future.

\section{Abbreviations}

lncRNAs: Long noncoding RNAs; CRC: colorectal cancer; EMT: epithelial-mesenchymal transition; CD: Crohn's disease; IBD: inflammatory bowel disease; COAD: Colon adenocarcinoma; ESCA: Esophageal carcinoma; GBM: Glioblastoma multiforme; HNSC: Head and Neck squamous cell carcinoma; KIRC: Kidney renal clear cell carcinoma; KIRP: Kidney renal papillary cell carcinoma; LIHC: Liver hepatocellular carcinoma; LUAD: Lung adenocarcinoma; LUSC: Lung squamous cell carcinoma; PAAD: Pancreatic adenocarcinoma; PCPG: Pheochromocytoma and Paraganglioma; READ: Rectum adenocarcinoma; STAD: Stomach adenocarcinoma; THCA: Thyroid carcinoma; UCEC: Uterine Corpus Endometrial Carcinoma; OS: overall survival; DFS: disease free survival.

\section{Supplementary Material}

Supplementary figures and tables. http://www.jcancer.org/v12p3909s1.pdf

\section{Acknowledgements}

This study was supported by Key Scientific Research Projects of Institutions of Higher Education in Henan Province (19A310024), The Medical Scientific and Technological Research Project of Henan Province (201702027), The National Natural Science Foundation of Henan Province (182300410342), The Health Commission Technology Talents Overseas Training Project of Henan Province (2018140) and The Key Scientific Research Project of Henan Higher Education Institutions (20A310024).

\section{Ethics approval and consent to participate}

The human cancer tissues used in this study were approved by Ethnics Committee of The First Affiliated Hospital of Zhengzhou University in December 19, 2019, and the TRN is 2019-KW-423.

\section{Competing Interests}

The authors have declared that no competing interest exists.

\section{References}

1. Huang $\mathrm{H}$, Liu $\mathrm{Y}$, Sung $\mathrm{T}$, Huang $\mathrm{T}$, Cheng $\mathrm{Y}$, Liou J, et al. TIMP3 expression associates with prognosis in colorectal cancer and its novel arylsulfonamide inducer, MPT0B390, inhibits tumor growth, metastasis and angiogenesis. Theranostics. 2019;9(22):6676-6689.

2. Siegel RL, Miller KD, Goding Sauer A, Fedewa SA, Butterly LF, Anderson JC, et al. Colorectal cancer statistics, 2020. CA Cancer J Clin. 2020;70(3):145-164.

3. Siveen K, Raza A, Ahmed E, Khan A, Prabhu K, Kuttikrishnan S, et al. The Role of Extracellular Vesicles as Modulators of the Tumor Microenvironment, Metastasis and Drug Resistance in Colorectal Cancer. Cancers. 2019;11(6). DOI: 10.3390/cancers11060746.

4. Luo K, Geng J, Zhang Q, Xu Y, Zhou X, Huang Z, et al. LncRNA CASC9 interacts with CPSF3 to regulate TGF- $\beta$ signaling in colorectal cancer. Journal of experimental \& clinical cancer research: CR. 2019;38(1):249. DOI:10.1186/s13046-019-1263-3.

5. Jung G, Hernández-Illán E, Moreira L, Balaguer F, Goel A. Epigenetics of colorectal cancer: biomarker and therapeutic potential. Nature reviews Gastroenterology \& hepatology. 2020;17(2):111-130.

6. Kapranov P, Cheng J, Dike S, Nix D, Duttagupta R, Willingham A, et al. RNA maps reveal new RNA classes and a possible function for pervasive transcription. Science (New York, NY). 2007:316(5830):1484-1488.

7. Salerno D, Chiodo L, Alfano V, Floriot O, Cottone G, Paturel A, et al. Hepatitis B protein HBx binds the DLEU2 IncRNA to sustain cccDNA and host cancer-related gene transcription. Gut. 2020;69(11):2016-2024.

8. Ma H, Chang H, Yang W, Lu Y, Hu J, Jin S. A novel IFNa-induced long noncoding RNA negatively regulates immunosuppression by interrupting H3K27 acetylation in head and neck squamous cell carcinoma. Molecular cancer. 2020;19(1):4. DOI: 10.1186/s12943019-1123-y.

9. Zhang Z, Weng W, Huang W, Wu B, Zhou Y, Zhang J, et al. A novel molecular-clinicopathologic nomogram to improve prognosis prediction of hepatocellular carcinoma. Aging (Albany N Y). 2020; 12(13):12896-12920.

10. Haberman $Y$, BenShoshan M, Di Segni A, Dexheimer P, Braun T, Weiss $B$, et al. Long ncRNA Landscape in the Ileum of Treatment-Naive Early-Onset Crohn Disease. Inflammatory bowel diseases. 2018;24(2):346-360

11. Wang $S$, Hou $Y$, Chen $W$, Wang J, Xie W, Zhang X, et al. KIF9-AS1, LINC01272 and DIO3OS IncRNAs as novel biomarkers for inflammatory bowel disease. Molecular medicine reports. 2018;17(2):2195-2202.

12. Leng X, Liu G, Wang S, Song J, Zhang W, Zhang X, et al. LINC01272 Promotes Migration and Invasion of Gastric Cancer Cells via EMT. OncoTargets and therapy. 2020;13:3401-3410.

13. Ulivi P, Scarpi E, Passardi A. Special Issue on Basic and Translational Research in Colorectal Cancer. International journal of molecular sciences. 2019;20(12). DOI: 10.3390/ijms20123095.

14. Hu Y, Jin Y, Wu X, Yang Y, Li Y, Li H, et al. LncRNA-HGBC stabilized by HuR promotes gallbladder cancer progression by regulating miR-502-3p/SET/AKT axis. Molecular cancer. 2019;18(1):167. DOI:10.1186/s12943-019-1097-9.

15. Wu N, Jiang M, Liu H, Chu Y, Wang D, Cao J, et al. LINC00941 promotes CRC metastasis through preventing SMAD4 protein degradation and activating the TGF- $\beta /$ SMAD2/3 signaling pathway. Cell death and differentiation. 2020. DOI: 10.1038/s41418-020-0596-y.

16. Gandhi M, Groß M, Holler J, Coggins S, Patil N, Leupold J, et al. The IncRNA lincNMR regulates nucleotide metabolism via a YBX1 - RRM2 axis in cancer. Nature communications. 2020;11(1):3214. DOI: 10.1038/s41467-020-17007-9.

17. Wang $\mathrm{W}$, Chen $\mathrm{T}$, Zeng $\mathrm{Z}$, Pan $\mathrm{Q}$, Huang $\mathrm{W}$, Han $\mathrm{C}$, et al. The lncRNA LAMP5-AS1 drives leukemia cell stemness by directly modulating DOT1L methyltransferase activity in MLL leukemia. Journal of hematology \& oncology. 2020;13(1):78. DOI: 10.1186/s13045020-00909-y.

18. Wang H, Wang $\mathrm{Y}$, Xie S, Liu Y, Xie Z. Global and cell-type specific properties of lincRNAs with ribosome occupancy. Nucleic acids research. 2017;45(5):2786-2796.

19. Ritter N, Ali T, Kopitchinski N, Schuster P, Beisaw A, Hendrix D, et al. The IncRNA Locus Handsdown Regulates Cardiac Gene Programs and Is Essential for Early Mouse Development. Developmental cell. 2019;50(5):644-657.e648

20. Sun Z, Ou C, Ren W, Xie X, Li X, Li G. Downregulation of long non-coding RNA ANRIL suppresses lymphangiogenesis and lymphatic metastasis in colorectal cancer. Oncotarget. 2016;7(30):47536-47555.

21. Sun Z, Ou C, Liu J, Chen C, Zhou Q, Yang S, et al. YAP1-induced MALAT1 promotes epithelial-mesenchymal transition and angiogenesis by sponging miR-126-5p in colorectal cancer. Oncogene. 2019;38(14):2627-2644. 
22. Sun L, Jiang C, Xu C, Xue H, Zhou H, Gu L, et al. Down-regulation of long non-coding RNA RP11-708H21.4 is associated with poor prognosis for colorectal cancer and promotes tumorigenesis through regulating AKT/mTOR pathway. Oncotarget. 2017;8(17):27929-27942.

23. Xiao G, Yao J, Kong D, Ye C, Chen R, Li L, et al. The Long Noncoding RNA TTTY15, Which Is Located on the Y Chromosome, Promotes Prostate Cancer Progression by Sponging let-7. European urology. 2019;76(3):315-326.

24. Chen C, He W, Huang J, Wang B, Li H, Cai Q, et al. LNMAT1 promotes lymphatic metastasis of bladder cancer via CCL2 dependent macrophage recruitment. Nature communications. 2018;9(1):3826. DOI: 10.1038/s41467-018-06152-x

25. Zhu P, Wu J, Wang $Y$, Zhu X, Lu T, Liu B, et al. LncGata6 maintains stemness of intestinal stem cells and promotes intestinal tumorigenesis. Nature cell biology. 2018;20(10):1134-1144.

26. Liu Y, Chang Y, Lu S, Xiang Y. Downregulation of long noncoding RNA DGCR5 contributes to the proliferation, migration, and invasion of cervical cancer by activating Wnt signaling pathway. Journal of cellular physiology. 2019;234(7):11662-11669. 